\title{
Epidemic analysis of the second-order transition in the Ziff-Gulari-Barshad surface-reaction model
}

\author{
Christopher A. Voigt and Robert M. Ziff \\ Department of Chemical Engineering, University of Michigan, Ann Arbor, MI 48109-2136
}

(September 13, 2021)

\begin{abstract}
We study the dynamic behavior of the Ziff-Gulari-Barshad (ZGB) irreversible surface-reaction model around its kinetic second-order phase transition, using both epidemic and poisoning-time analyses. We find that the critical point is given by $p_{1}=0.3873682 \pm 0.0000015$, which is lower than the previous value. We also obtain precise values of the dynamical critical exponents $z, \delta$, and $\eta$ which provide further numerical evidence that this transition is in the same universality class as directed percolation.
\end{abstract}

PACS numbers(s): 05.70.Jk, 11.25.Hf, 64.60.Ak

There has been a great deal of interest surrounding the critical behavior of non-equilibrium kinetic models, including directed percolation (DP) [1,2], the contact process [3] and various surface-reaction or catalysis models (for review see (⿶) ). These models all contain a similar continuous (second-order) phase transition from an adsorbing to a vacuum state [5,6].

It has been shown that many of these transitions behave in a universal manner even though the systems abide by different local rules and are inherently modeling different physical systems. Grassberger [i] and Janssen [8] postulated that all single-component continuous transitions fall into the robust DP or "Reggeon field theory" class, and many numerical simulations have supported this hypothesis (i.e., [6, 7.,9]). Grinstein et al. [5] were the first to hypothesize that the specific oxygen-poisoning (second-order) transition of the ZGB surface reaction model [11 falls into this class of models, and Jensen et al. ran simulations which support this conclusion [7]. In this communication, we report on new, very extensive simulations which provide further support for this hypothesis, and correct an apparent error in the reported value of the location of that transition. Assuming the identification of the ZGB model with the DP class to be exact, our results give the most accurate values of the DP dynamical critical exponents to date.

The ZGB model is a simplified model for the irreversible reaction of $\mathrm{CO}(\mathrm{A})$ and $\mathrm{O}_{2}\left(\mathrm{~B}_{2}\right)$ catalytic reaction on a $\mathrm{Pt}$ surface. The simulation involves the adsorption and reaction of species on a square lattice and proceeds via the Langmuir-Hinchelwood mechanism, in which all molecules must adsorb before they can react. The following kinetic scheme is employed,

$$
\begin{aligned}
& A+* \rightarrow A^{*} \\
& B_{2}+* \rightarrow 2 B^{*} \\
& A^{*}+B^{*} \rightarrow A B+2 *
\end{aligned}
$$

where ${ }^{*}$ refers to a lattice site. A Monte-Carlo algorithm is employed where a site is randomly chosen. If the site is empty, an A will adsorb with probability $p$. With probability $1-p$, a $\mathrm{B}_{2}$ is adsorbed and instantly dissociates onto that site and a randomly chosen neighboring site if the latter is empty. When a species adsorbs, it checks for adjacent neighbors of the opposite species. If one is present, the two react immediately, implying an infinite reaction rate as compared to the adsorption rate. There are two transition points in this system. At $p_{2}$, there is a first-order (discontinuous) transition to an A-poisoned (saturated) state, and at $p_{1}$ there is a second-order (continuous) transition to a B-poisoned state. Between these points exists a reactive window where the system can reach a steady state and react indefinitely. (For a phase diagram, see [11].) Even within the window, for finite systems, the system is only metastable as it can in principle poison by a statistical fluctuation to a non-reactive state. However, here the average time to poison $t_{p}$ grows exponentially with lattice size $L$, the signature of a reactive steady state [12]. The value of $p_{2}$ has been accurately determined to be $0.52560 \pm 0.00001$ [13] using the constant-coverage ensemble algorithm. This algorithm, however, is only applicable to finding the location of the first-order transition.

Because the second-order transition is a continuous one to a single adsorbing state, it is expected to fall into the DP class [6]. Indeed, while the ZGB model involves three components (A, B, and vacant sites), at the second-order transition, there are rarely A molecules at the surface so it is essentially a two-species model like other members of the DP class. The value of its transition point $p_{1}$ was first empirically observed to be $0.389 \pm 0.005$ [11]. A more precise value $0.39065 \pm 0.00010$ was obtained by Jensen et al. by using an epidemic analysis [7]. However, while recently performing some other investigations [13], we found that this value appears to be somewhat high. Thus, we carried out new simulations, using the epidemic procedure as well as a poisoning-time analysis, to re-examine the value of $p_{1}$ and the related dynamic critical exponents. 
The epidemic method was initially used to study the contact process [3] and has been successfully applied to determine the critical exponents and the critical point for DP [1]. To run the epidemic analysis, we started with a large $(1024 \times 1024)$ system completely saturated with B except for a single vacant site in the center. A large system is necessary so that the cluster never hits the boundary. The simulation was run at a set value of $p$ and a reactive cluster was grown and watched until the system reverted to a non-reactive adsorbate state, or a maximum cutoff time was reached.

The vacant sites (numbering $n_{v}$ ) were kept on a list which was randomly accessed for each adsorption trial, incrementing the time $t$ by $1 / n_{v}$. As each cluster grew, the quantities of interest were recorded in $\log _{2}$ bins of time. Since only approximately $3 \%$ of all clusters grown reached the last bin, it was necessary to make numerous runs order to obtain satisfactory statistics. For the values $p=0.38736$ and $0.38737,8 \times 10^{7}$ clusters $(N)$ were grown up to $2^{13}=8192$ time-steps, requiring a total of 200 days of computational time on a HP 9000 series UNIX platform. In the work of [7] in contrast, only 100000 to 250000 clusters were grown up to 1000 timesteps. Although we could pinpoint $p_{1}$ to four significant figures in just a few hours, we decided to carry out extensive runs in order to find $p_{1}$ to six significant digits and to determine the dynamical critical exponents precisely.

We measured the three quantities introduced by Grassberger and de la Torre [3]: the survival probability $P(t)$, the mean number of vacancies (averaged over $N) n(t)$, and the mean-square radius of gyration of vacant sites (averaged over $N$ alive at $t), R^{2}(t)$. At the critical point, these are hypothesized to follow the asymptotic power laws,

$$
\begin{aligned}
P(t) & \sim t^{-\delta} \\
n(t) & \sim t^{\eta} \\
R^{2}(t) & \sim t^{z}
\end{aligned}
$$

These exponents follow the hyperscaling relation [3]

$$
d z=2 \eta+4 \delta
$$

where $d$ is the spatial dimension. These relationships provide a powerful method to determine $p_{1}$ by evaluating the effects of slightly non-critical values of $p$, in which case the resulting behavior deviates from (何) - (6) for large $t$. An example of this is shown in Fig. 1 where $n(t)$ is plotted for $p=0.39065,0.3873682$, and 0.38407 . The upper curve is for the value of $p_{1}$ reported in [7], while the central curve is for the value we find below.

In order to find the exponents accurately, we consider the local slopes which are defined as

$$
-\delta(t)=\ln [P(t) / P(t / 2)] / \ln 2
$$

and similarly for $\eta(t)$ and $z(t)$. (Here we used a factor of 2 rather than 5 or 8 of previous work [2,7], which we could do because of our higher statistics.) These are all graphed in Fig. 2 for $p=0.38736$ and 0.38737 . The local slopes can be expanded as [3]

$$
\delta(t)=\delta+\frac{a}{t}+\frac{b}{t^{\delta^{\prime}}}
$$

If the non-analytic corrections were negligible, then it would be easy to extrapolate the critical exponents as a function of $1 / t$ as discussed by Grassberger. However, these corrections are rather large and therefore hinder a direct linear extrapolation. In order to overcome this problem, we grew over $5 \cdot 10^{8}$ clusters to $2^{9}$ time-steps so that we could better follow the non-analytical trajectory of each curve. Extrapolating these results to $t \rightarrow \infty$, we find

$$
\delta=0.4505 \pm 0.001, \eta=0.2295 \pm 0.001, z=1.1325 \pm 0.001 .
$$

consistent with $\delta=0.452 \pm 0.008, \eta=0.224 \pm 0.10$ and $z=1.133 \pm 0.002$ found in [7].

For comparison, the updated values recently found by Grassberger and Zhang [2] for DP are

$$
\delta=0.451 \pm 0.003, \eta=0.229 \pm 0.003, z=1.133 \pm 0.002 .
$$

The precise agreement between (10) and (11) leaves little doubt that the ZGB model is included in the DP class as predicted by Grinstein et al. [5].

For $p$ away from $p_{1}, n(t)$ follows the scaling behavior [3]

$$
n(t) \sim t^{-\eta} \phi\left[\left(p-p_{1}\right) t^{1 / \nu_{\|}}\right]
$$

and similarly for $P(t)$ and $R^{2}(t)$. It follows from this equation that 


$$
\left.\frac{d \ln n}{d \ln p}\right|_{p=p_{1}} \propto t^{1 / \nu_{\|}}
$$

where $\nu_{\|}$is the time-domain correlation length exponent. In Fig. 3, we plot the quantity on the left-hand side of the equation above, calculated by taking the difference of $n(t)$ for $p=0.38736$ and 0.38737 , vs. $\ln t$. This plot shows that Grassberger's value for DP $\nu_{\|}=1.295 \pm 0.006$ [2] is completely consistent with our data.

To determine the precise value of $p_{1}$, we expand the scaling function $\phi$ as

$$
P(t) \approx t^{-\delta}\left[a+b\left(p-p_{1}\right) t^{1 / \nu_{\|}} \ldots\right]
$$

This equation implies that a plot of $P(t) t^{\delta}$ vs. $t^{1 / \nu_{\|}}$for values $p$ close to $p_{1}$ should yield straight lines and that a direct linear interpolation of the data from different values of $p$ can be used to find $p_{1}$ (which corresponds to a horizontal line in this plot) (Fig. 4). There is an initial curvature which is to be expected for small clusters due to finite-cluster effects. To minimize this effect, we have added a constant $c$ to the time that effectively allows for a higher-order analytic correction term:

$$
(t+c)^{-\delta} \approx t^{-\delta}\left(1-\frac{\delta c}{t}\right)
$$

where $c \approx 1.7$ was found to give the best results. The resulting plot of our data is shown in Fig. 4 . The statistical fluctuations in each bin are given by

$$
\sqrt{\frac{N_{\text {bin }}\left(N_{\text {total }}-N_{\text {bin }}\right)}{N_{\text {total }}}}
$$

which implies that the largest bins which have the most accurate data also have the greatest error (least precision). Interpolating the two data curves in Fig. 4 , we deduce that $p_{1}$ is given by

$$
p_{1}=0.3873682 \pm 0.0000015 \text {. }
$$

This result is nearly two orders of magnitude more precise than the result of [7], $0.39065 \pm 0.00010$, and more than 30 combined error bars lower. We believe that some error must have occurred in the simulations or analysis of [7].

To confirm our value for $p_{1}$, we also ran a poisoning-time analysis [13] of the system at its critical point. Similar methods have been applied to other problems including the quantification of finite lattice effects [12,8, 14. To do this, we essentially run the opposite dynamic algorithm performed by the epidemic analysis. We start with a small lattice in a fully reactive state (all vacant sites) and set the value of $p$ at our determined $p_{1}$. Periodic boundary conditions are applied and the system is allowed to run until the adsorbate B saturates or poisons the system, causing a global non-reactive state. When the value of $p$ is at $p_{1}$, it is expected that the dependence of $t_{p}$ on $L$ will be power-law, and when $p \neq p_{1}$, the dependence will be exponential 12, 14. We ran this simulation for square lattices of powers of 2 in size from $8 \times 8$ to $64 \times 64$ for roughly $10^{5}$ runs each. Here, a time-step is defined as $L^{2}$ adsorption trials. Fig. 5 shows the results of our analysis and it was found that at $p_{1}$, the relationship is indeed

$$
t_{p} \sim L^{w}
$$

with $w=1.77 \pm 0.02$. In [13], we observed that $w=2 / z=\nu_{\|} / \nu_{\perp}$, indicating that the time to expand a reactive state scales as the time to contract. The $z$ implied by this result is consistent with the value determined above. While this method is evidently less efficient than the epidemic analysis, it provides a useful confirmation our results for $p_{1}$ and $z$.

In conclusion, we have provided improved numerical evidence that the ZGB oxygen-poisoning transition falls into the larger DP class of nonequilibrium models. Accepting that that hypothesis is true, which seems certain, our exponents represent the most accurate values of the DP dynamic critical exponents to date (by a factor of about two). We also independently confirm that the value of $\nu_{\|}$for the ZGB model falls into the DP class and use it to find a highly accurate and corrected value of $p_{1}$.

This material is based upon work supported by the US National Science Foundation under Grant No. DMR-9520700.

[1] P. Grassberger, J. Phys. A: Math. Gen. 22, 3673 (1989). 
[2] P. Grassberger and Y. Zhang, Physica A 224, 169 (1996).

[3] P. Grassberger and A. de la Torre, Ann. Phys. (N.Y.) 122, 373 (1979).

[4] E. V. Albano, Heterogeneous Chemistry Reviews 3, 389 (1997).

[5] G. Grinstein, Z. W. Lai and D. A. Browne, Phys. Rev. A 40, 4820 (1989).

[6] P. Grassberger, Z. Phys. B 47, 365 (1982).

[7] I. Jensen, H. Fogedby and R. Dickman, Phys. Rev. A 41, 3411 (1990).

[8] H. K. Janssen, Z. Phys. B 42, 151 (1981).

[9] P. Meakin and D. Scalapino, J. Chem. Phys. 86, 731 (1987).

[10] R. M. Ziff and B. Brosilow, Phys. Rev. A 46, 4630 (1992).

[11] R. M. Ziff, E. Gulari and Y. Barshad, Phys. Rev. Lett. 56, 2553 (1986).

[12] D. ben-Avraham, S. Redner, D. B. Considine and P. Meakin, J. Phys. A: Math. Gen. 23, L613 (1990).

[13] C. A. Voigt and R. M. Ziff, to be published.

[14] R. Dickman and A. Moreira, e-print //xxx.lanl.gov/archive/cond-mat/9709082

FIG. 1. The behavior of the number of vacant sites $n$ as plotted against time $t$, for $p=0.39065,0.3873682$, and 0.38407 (top to bottom).

The upper value is $p_{1}$ from [7], and the center is for the value found here.

FIG. 2. The three critical exponents derived from our epidemic analysis: $\delta$ (a), $\eta$ (b), and $z$ (c). These values show super-critical $(\diamond: p=0.38737)$ and sub-critical $(\triangle: p=0.38736)$ behavior. Each of these lines represents the average of $3.5 \times 10^{7}$ runs. The actual value of $p_{1}$ falls between these lines and can be determined by linear interpolation as in Fig. 4 .

FIG. 3. Plot to determine $\nu_{\|}$from (13) by using the values of $p=0.38736$ and 0.38737 . The line is for $\nu_{\|}=1.295$ as determined by Grassberger for DP. It can be seen that the ZGB data is consistent with this value.

FIG. 4. Plot allowing a linear interpolation for $p_{1}$ as expressed in equation (14). The lines for $0.38736(\triangle)$ and 0.38737 (०) represent the sub- and super-critical behavior respectively. The bold line represents the interpolation for the value, $p_{1}=0.3873682$. The error bars were calculated as in (16). Here, $t$ is offset by an additive constant 1.7 to improve small-time behavior.

FIG. 5. Results of our poisoning-time analysis for the same values of $p$ displayed in Fig. $1(\triangle: 0.39065, \circ: 0.3873682, \diamond$ : $0.38407)$. This plot demonstrates that the expected power-law behavior obtains when our value of $p_{1}$ is used. 
Fig. 1

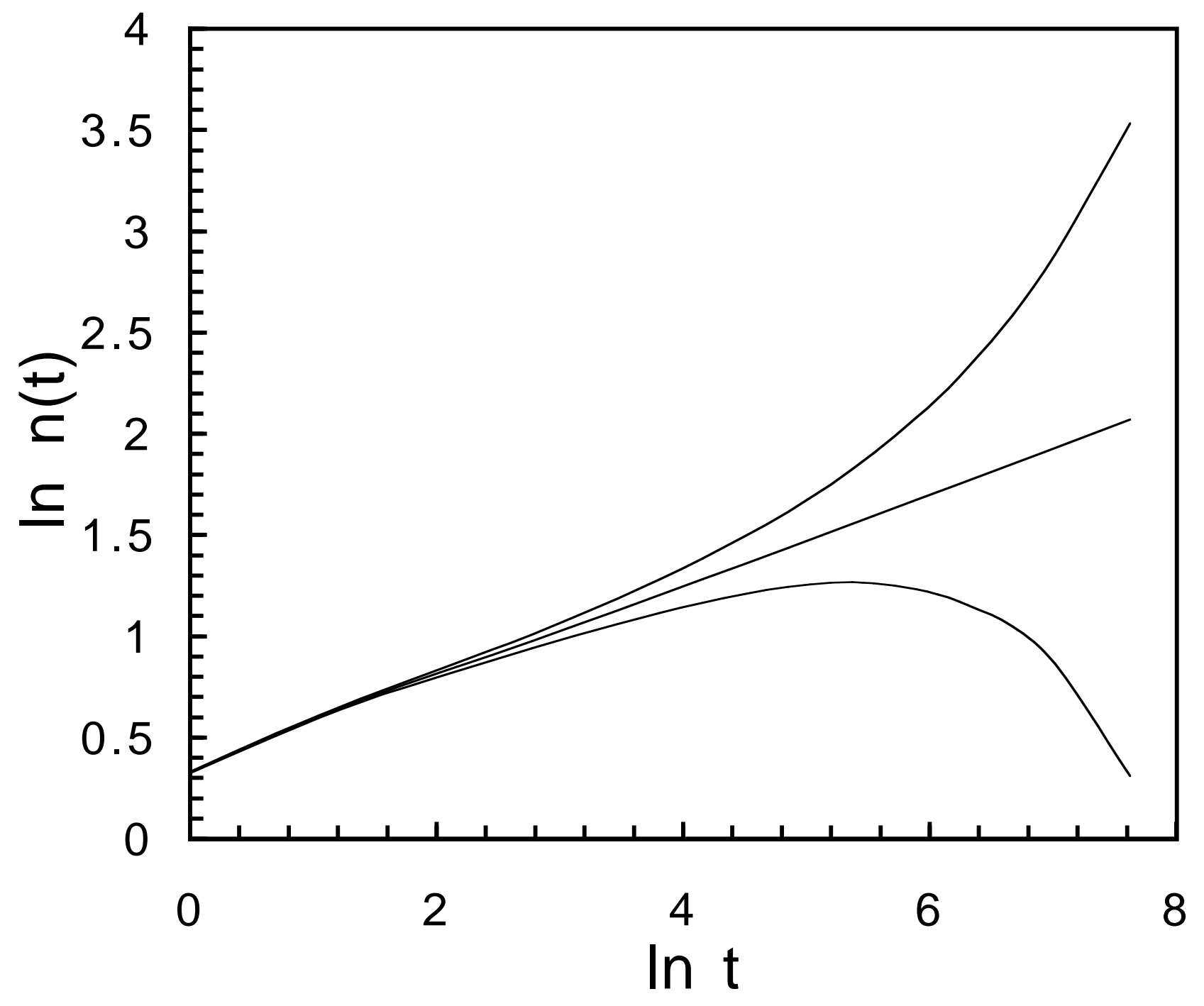


Fig. 2 (a)

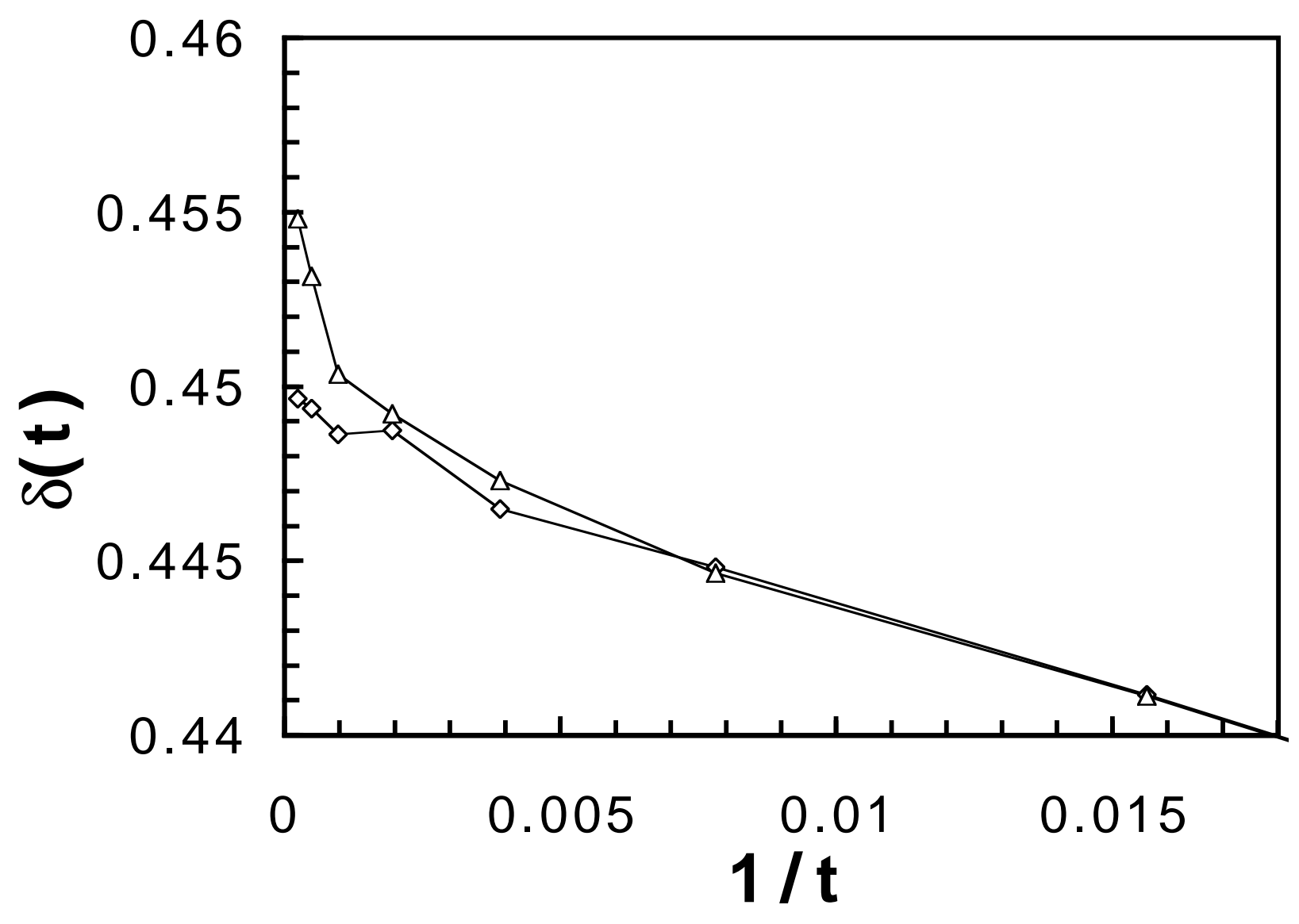


Fig. 2 (b)

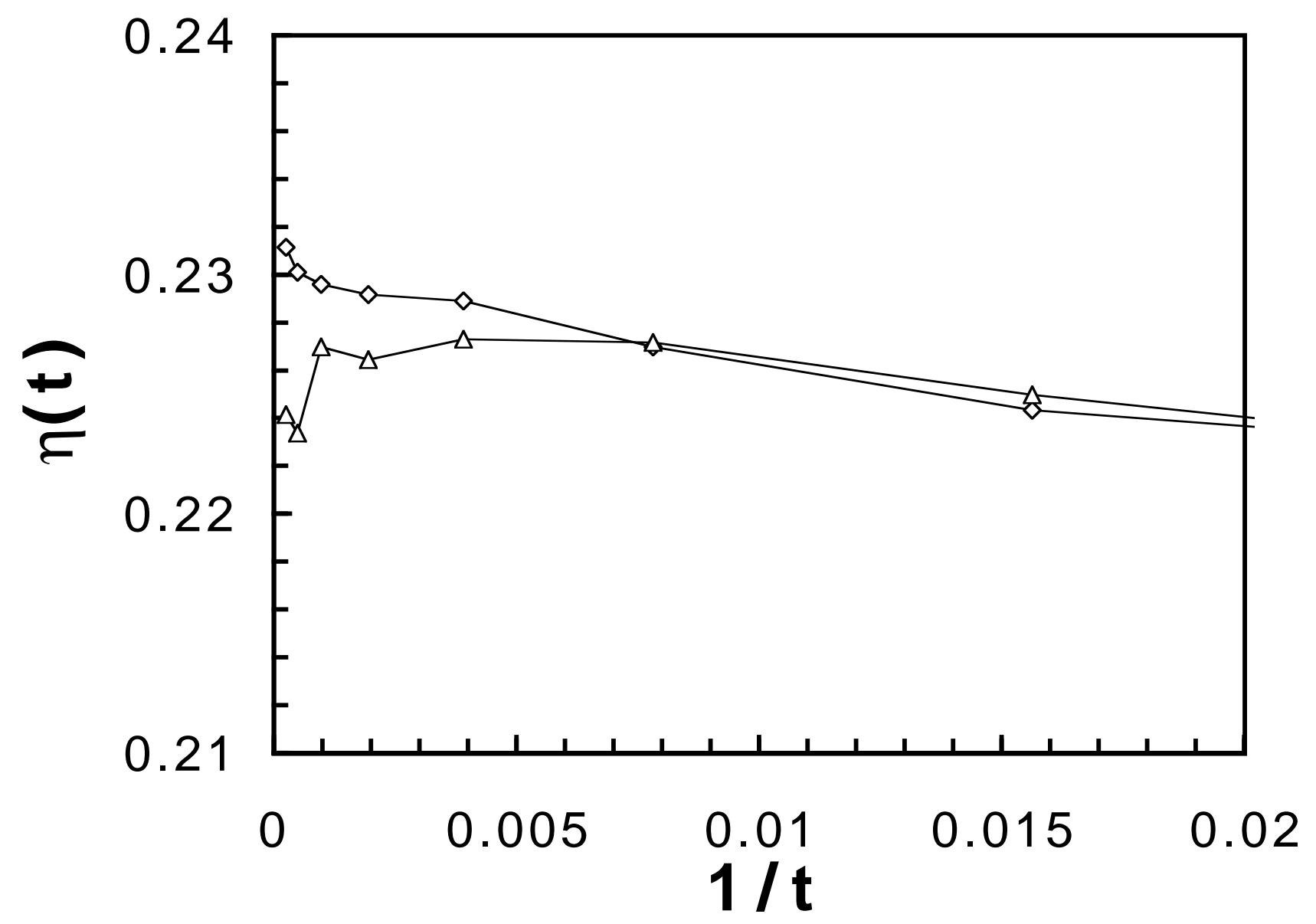


Fig. 3




Fig. 4

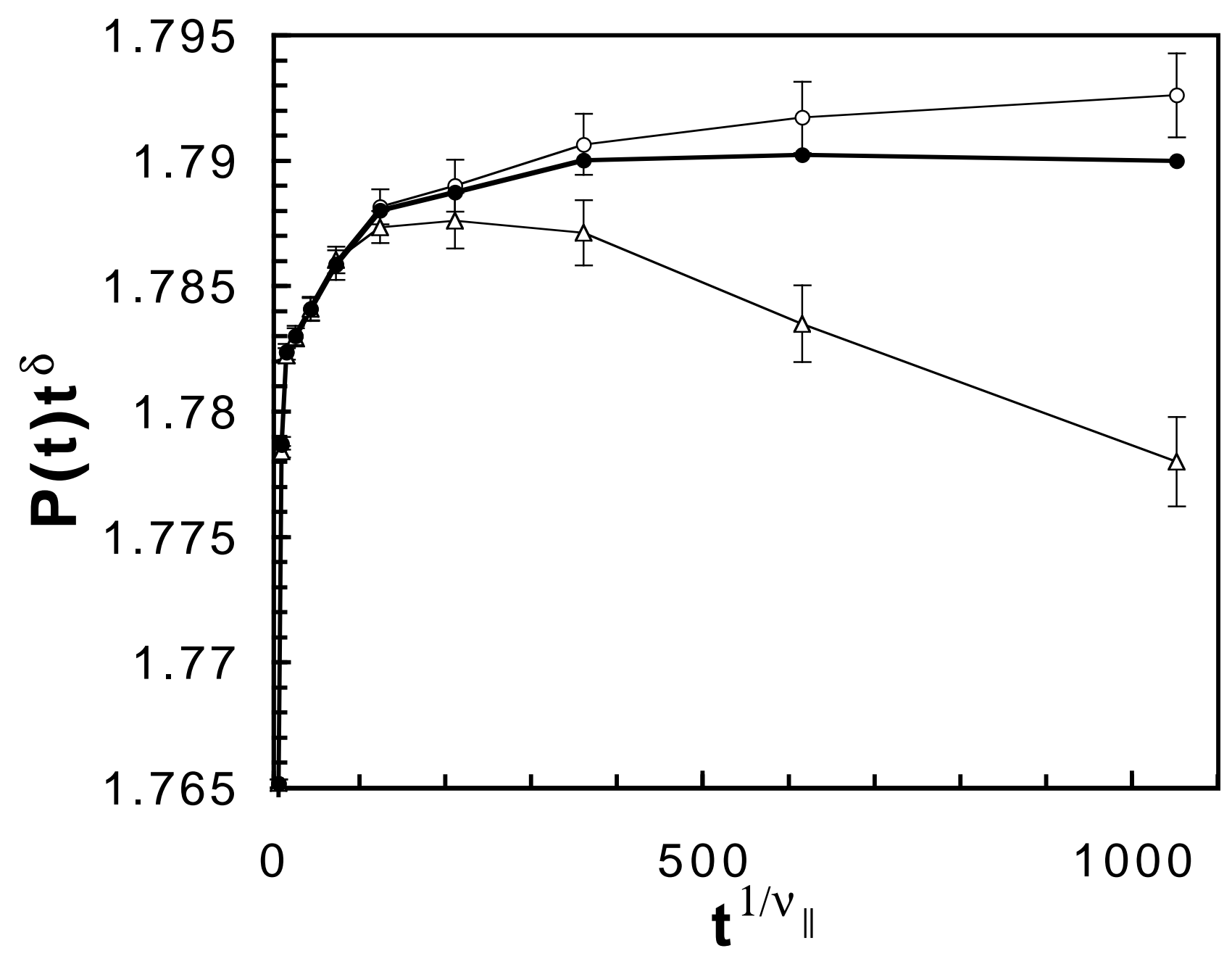


Fig. 5

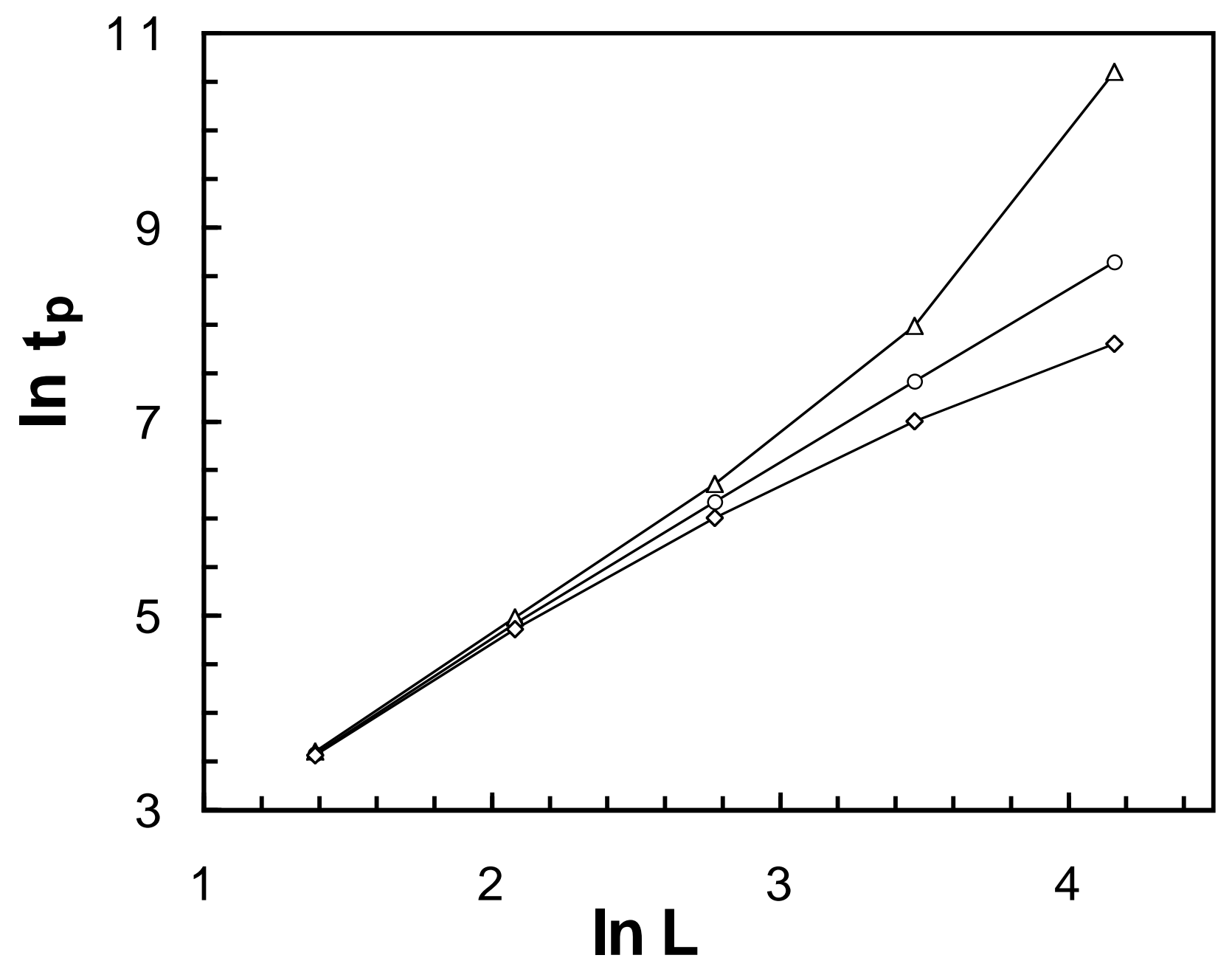

